Recepción: 10 / 04 / 2018

Aceptación: 06 / 07 / 2018

Publicación: 15 / 09 / 2018

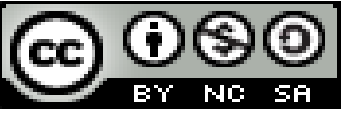

Ciencias de la educación Artículo de investigación

\title{
Educación ambiental en la educación superior. Una mirada desde el currículo
}

\author{
Environmental education in higher education. A look from the curriculum
}

Educação ambiental no ensino superior. Um olhar do currículo

Margarita P. Luque-Espinoza de los Monteros ${ }^{\mathrm{I}}$

mluque@umet.edu.ec

\section{Correspondencia: mluque@umet.edu.ec}

I Magister en Educación Superior, Doctora en Ciencias de la Educación, Especialización Psicología Educativa, Licenciada en Psicología Educativa, Psicólogo Educativo, Universidad Metropolitana Matriz Guayaquil, Guayaquil, Ecuador. 


\section{Resumen}

El presente trabajo de investigación, tiene como objetivo hacer una revisión y análisis de diversos trabajos relacionados con la temática de investigación, los cuales han hecho aportaciones significativas al tema de la educación ambiental en la educación superior.

El objetivo principal es proponer un nuevo programa de estudio de Educación Ambiental, que incorpore la protección del medio ambiente que promueva un desarrollo social y sostenible de tipo inclusivo, donde se resalte la dignidad del ser humano.

Actualmente existen investigaciones en Latinoamérica, que aportan argumentos conceptuales suficientes para derivar los criterios con los cuales se ha realizado la formación ambiental en la educación superior en los últimos 15 años, este ha sido un motivo para la autora tomar como base y modelo de ponerlo en práctica en la Universidad Metropolitana. Los métodos empleados para la revisión y análisis de investigaciones: ámbito pedagógico, didáctico y programas de estudio de asignatura de educación ambiental. Como resultados diversas experiencias enriquecedoras orientadas a la innovación curricular, son muchos los procesos sin tendencias claras de una visión de educación ambiental, currículos como planes de estudio de educación ambiental centrados en lo teórico, por lo cual surge una propuesta de formación ambiental universitaria de estilo teóricopráctico.

Palabras clave: educación ambiental; educación superior; currículo.

\section{Abstract}

The aim of this research work was to review and analyze various works related to the research topic, which have made significant contributions to the topic of environmental education in higher education. The main objective was to establish new adjustments to the study program of Environmental Education, which incorporates the protection of the environment that promotes an inclusive social and sustainable development, where the dignity of the human being is highlighted. There are currently investigations in Latin America, which provide enough conceptual arguments to derive the criteria with which environmental education has been conducted in higher education in the last 15 years, this was a reason for the author to take as a basis and model to put it into practice at the Metropolitan University. The methods used for the review and analysis of research: pedagogical, didactic field and study programs of environmental education subject. As results diverse enriching experiences oriented to the curricular innovation, 
are many the processes without clear tendencies of a vision of environmental education, curricula like plans of study of environmental education centered in the theoretical thing, for which a proposal of university environmental formation of style arises theoretical-practical

Keywords: environmental education; higher education; curriculum.

\section{Resumo}

O objetivo deste trabalho de pesquisa é revisar e analisar diversos trabalhos relacionados ao tema da pesquisa, os quais contribuíram significativamente para o tema educação ambiental no ensino superior.

O objetivo principal é propor um novo programa de estudos de Educação Ambiental, que incorpore a proteção do meio ambiente que promova um desenvolvimento social e sustentável de tipo inclusivo, onde a dignidade do ser humano seja destacada.

Atualmente há uma pesquisa na América Latina, que fornecem argumentos conceituais suficientes para derivar critérios que foram realizados formação ambiental no ensino superior ao longo dos últimos 15 anos, esta tem sido uma razão para o autor para construir e modelá-lo em prática na Universidade Metropolitana. Os métodos utilizados para a revisão e análise da pesquisa: campo pedagógico, didático e programas de estudo da disciplina de educação ambiental. Como resultado de muitas experiências enriquecedoras inovação curricular orientada, muitos processos, sem tendências claras de uma visão de currículos de educação ambiental e programas de educação ambiental focada no teórico, por isso uma proposta de estilo faculdade formação ambiental emerge teórico-prático.

Palavras chave: educação ambiental; Educação superior; currículo.

\section{Introducción}

La Universidad Metropolitana, fundamenta su quehacer a través de la docencia y la investigación, teniendo en cuenta que uno de los procesos fundamentales para alcanzar el desarrollo sustentable y sostenible del país, es mediante la educación ambiental, como herramienta que juega un papel preponderante para llevar a una sociedad hacia la sostenibilidad.

La formación ambiental fue concebida desde la Conferencia de Tbilisi como el proceso de construcción de un saber interdisciplinario y de nuevos métodos holísticos para analizar los 
complejos procesos socios ambientales que emergen del cambio global (Organización de las Naciones Unidas para la Educación, 1994). Durante siglos, la especie humana ha transformado el entorno en que vive en busca de satisfacer sus necesidades, corrompiendo la relación sociedad medio ambiente, ocasionando a la vez una contable pérdida en valores, desarrollando normas de uso indiscriminado que junto a los avances de la ciencia y tecnología le han concedido al hombre poder sobre el entorno, ocasionando una acción depredadora de este sobre el medio, originando cambios en las condiciones de vida del planeta, efectos nocivos y depredadores que afectan la calidad de la vida de todos los seres vivos.

En la práctica educativa aun cuando se han dado avances en el cuidado y protección del medio ambiente, aspecto tratado con mayor profundidad en algunas carreras y asignaturas que guardan relación directa con el tema, los resultados de encuestas a docentes y entrevistas a directivos permiten expresar como principales insuficiencias que inciden en la formación de una cultura ambiental las siguientes: a) Prevalece en ocasiones una concepción teórico- metodológica restringida de la transversalidad de la temática para ser abordada en la gestión educativa. b) Los programas de estudio de las carreras y asignaturas no cuentan con la actualización de contenidos que respondan a la educación ambiental para el desarrollo sostenible, desde una perspectiva comunitaria y participativa. c) Es limitada la gestión de proyectos de investigación, proyectos de vinculación con la sociedad y la capacitación docente en temas relacionados con el cuidado y protección del medio ambiente.

Por ello el currículo universitario debe permitir una conexión entre la teoría y la práctica educativa a nivel institucional, que redimensione esta relación con la formación de estudiantes comprometidos con la sociedad, mediante la labor docente trabajando actividades o programas de educación ambiental para el desarrollo sostenible.

El presente trabajo de investigación, tiene tres enfoques: Educación ambiental, Educación superior y Currículo. La metodología utilizada es un estudio descriptivo y de carácter exploratorio, se realiza un análisis documental, utilizados para recabar de la información aplicando técnicas a través del análisis de investigaciones y documentos de la Universidad Metropolitana. 


\section{Importancia del problema}

A nivel mundial, el deterioro de los recursos naturales, sufrido por el planeta, ha llegado a afectar en todo sentido, en mayor o menor grado la vida de las personas, influyendo en sus conductas, actitudes y comportamientos, todo ello lleva a que se investigue desde hace más de 4 décadas sobre los problemas ambientales.

La situación de pobreza que va en incremento, es un factor preocupante y a la vez cuestionador, debido a que producto de ello surgen situaciones de violencia y problemáticas ambientales, relacionadas con la lógica del capital que prioriza una racionalidad egoísta y ambiciosa en la cual el hombre no cuenta sino como un simple número impersonal de las encuestas e indicadores económicos, llegando a valorizar lo económico y material y degradando al ser humano, reduciéndolo a una simple estadística.

En los actuales momentos alrededor del consumismo existente, se tejen unas experiencias basadas en la ansiedad por el cambio constante y vertiginoso de las vivencias humanas. Por ello (Maya. A., 2003), plantea que la crisis ambiental no es un problema ecosistémico, sino fundamentalmente, un problema cultural, que aunque repercute sobre lo ecosistémico, "se origina en la forma organizativa que ha adquirido la especie humana".

Ante esta crisis, se debe buscar una solución que se alimente de un ejercicio crítico y reflexivo, pues como lo recuerda (Vega. R, 1999), "nuestra obligación es ayudar a que se mantenga viva la capacidad de las nuevas generaciones para razonar, preguntar y criticar, mientras, entre todos, reconstruimos los programas para una nueva esperanza".

De todas las conceptualizaciones que se realicen sobre lo ambiental, dependerá el grado de comprensión del fenómeno o problema que, en principio, debe estar en pro de una ética del respeto a la vida en todas sus manifestaciones.

El primero en emplear la frase "educación ambiental fue el Dr. William Stapp, profesor de la Universidad de Michigan en 1969. Además de concienciar a la población a través de la educación, muchos gobiernos buscan solución a la agresión ambiental con las energías alternativas, las cuales aprovechan los factores ambientales y no crean alteraciones de medio. 
Posteriormente, esta orientación inicial fue reiterada por la Conferencia Mundial sobre Educación y Formación Ambiental (UNESCO-PNUMA, 1987), así como por el Programa 21, emanado de la (ONU, 1992).

La incorporación de los aspectos ambientales en los currículos de las carreras universitarias tradicionales, particularmente las siguientes: derecho, economía, medicina, arquitectura y urbanismo, educación, agronomía, ciencias biológicas y naturales.

La capacitación en las carreras o profesiones que se requieren para la protección, rehabilitación y ordenación del medio ambiente.

La realización de programas de formación de personal docente universitario en la esfera del medio ambiente.

Tal como lo refiere (Novo. M, 1995) en la revista Iberoamericana de Educación: "El avance más importante quizá sea que ahora se generaliza al fin la comprensión de que la problemática ambiental es un fenómeno global y comienza a percibirse esta idea de globalidad aparejada la idea de relación, y de interrelaciones entre los problemas y entre los fenómenos ambientales".

Es también esencial que los medios de comunicación eviten contribuir al deterioro del medio humano y difundan información de carácter educativo sobre la necesidad de protegerlo y mejorarlo, a fin de que el hombre pueda desarrollarse en todos los aspectos.

En el caso específico de Ecuador, la (República. del. Ecuador, 2008) proclama que son deberes primordiales del Estado defender el patrimonio natural y cultural del país y proteger el medio ambiente y preservar el crecimiento sustentable de la economía, y el desarrollo equilibrado y equitativo en beneficio colectivo (entre otros). Se instituye en las secciones de: Educación en el Artículo 27 se establece que: "La educación se centrará en el ser humano y garantizará su desarrollo holístico, en el marco del respeto a los derechos humanos, al medio ambiente sustentable". Ciencia y Tecnología - En el Artículo 80 establece que: "el Estado fomentará la ciencia y la tecnología, especialmente en todos los niveles educativos, dirigidas a mejorar la productividad, la competitividad, el manejo sustentable de los recursos naturales, y a satisfacer las necesidades básicas de la población". 
El sujeto investigador, es participante activo durante todas las fases del proceso investigativo, puesto que "los roles que van desempeñando el investigador y los elementos de la unidad social objeto de estudio son fruto de una definición y negociación progresiva" (García. E., 1996)

Por ello, esta investigación se enmarca en la relación subjetiva entre la investigadora y la problemática abordada. Relación que, defendida por el llamado paradigma cualitativo, se alimenta continuamente de la confrontación permanente de las realidades intersubjetivas que emergen a través de la interacción del investigador con los actores de los procesos y realidades socio-culturales y personales objeto de análisis, así como, del análisis de la documentación teórico, pertinente y disponible”. (Tamayo. M., 1999)

Es importante que las universidades estén preparadas para asumir los retos que impone una verdadera educación ambiental, para ello, la participación de los miembros involucrados en las dinámicas institucionales es vital, no solo para producir el conocimiento relacionado con la problemática, sino también para comprender de manera conjunta los problemas ambientales presentes en el contexto en el que se encuentran. Debe existir un dialogo que provea a la universidad de los elementos necesarios para comenzar a instaurar una reflexión crítica en torno a lo educativo en materia ambiental.

El fomento y desarrollo de una cultura que evidencia una conciencia ciudadana y planetaria para la conservación, defensa y mejoramiento del ambiente; para el logro de una vida sana; para el uso racional, sostenible y sustentable de los recursos naturales. En tal sentido, las instituciones de educación superior deben promover y crear eventos para sensibilizar a los diferentes agentes educativos de la magnitud de los problemas que afectan al medio ambiente en que se desarrolla nuestra existencia, así como movilizar un actuar hacia acciones responsables de cuidado y protección del medio ambiente y por ende cultura vinculados a eventos oficiales.

Es por ello que se debe antes de iniciar cualquier programa, fortalecer la consciencia de los participantes, con una articulación de un currículo que responda y contribuya a mantener una unión entre la cultura, la sociedad y la naturaleza. El rol que cumpla el formador juega un papel preponderante en el campo de lo ambiental, de esta manera ellos son quienes ayudarán a poder enfrentarse al medio social, de esta manera se podría consolidar el poner término a la concepción 
capitalista del medio ambiente que según (Ibarra. G, 1998) "no cuestiona el modelo económico de corte neoliberal, ni la relación de la explotación que establece el hombre con la naturaleza".

En la actualidad lo que es más palpable a nuestros ojos en cuanto a necesidad de trabajar en temas ambientales tenemos que:

Falta de conciencia y amor por el medio que nos rodea: naturaleza y recursos.

Desinformación sobre las consecuencias ambientales a nivel global, regional y local de la mala administración del medio ambiente y el uso inadecuado de los recursos naturales.

Falta de conciencia sobre el buen aprovechamiento de los recursos naturales.

Desinterés por el buen manejo de los residuos sólidos en todas las áreas físicas.

Haciendo un poco de historia en el campo educativo formal, durante muchos años, fue necesario superar la predisposición de la tradición educativa de fraccionar los aprendizajes, en esta línea la tarea consistió en convencer, también desde las bases, a las autoridades educativas, de que la educación ambiental, tenía que ser una dimensión que impregnara todo el currículo.

En la década de los setenta se dieron los primeros pasos, las primeras experiencias en las que el medio ambiente era considerado, como un centro de interés y en las que intervenían profesores de diversas asignaturas, estos trabajos fueron el cimiento de toda la comprensión de un nuevo método de acercamiento a la realidad, que posteriormente se desarrollaría en esta línea.

Es necesario considerar que este período fue considerado como una década difícil para afianzar algo que hoy está bastante asumido, afortunadamente, y que la educación ambiental, es algo más que un movimiento ético, aunque ha sido difícil consolidar este movimiento que nació de la tradición conservacionista, pero que ha tenido la capacidad de permitir que se avance más allá del simple conservacionismo, es la época en que empieza a divulgarse más allá del mundo científico todo el problema de la capa de ozono, de los cambios climáticos.

En los actuales momentos, una manera de abordar la problemática ambiental, es mediante un proceso de formación basada en la ética, que como señala (Bermúdez. O, 2005) no esté "limitada al ser humano y sus actos, sino que trasciende la relación que se establece entre la sociedad y la naturaleza, entre los ecosistemas y las culturas". 
Se debe fomentar el desarrollo de una educación ambiental en directivos, docentes, estudiantes y trabajadores para comprometer a toda la colectividad universitaria en el cumplimiento de los objetivos y metas ambientales que se propongan y para concientizar acerca de la necesidad de proteger el medio ambiente con un enfoque de desarrollo sostenible constituye hoy más que nunca una tarea impostergable que debe ser atendida desde sus procesos sustantivos. Esta situación es posible si se lo enfoca con una perspectiva reflexiva y crítica desde el campo del currículo, en el que exista una interrelación entre los contextos educativos, culturales y sociales. Debido a que los cambios ambientales se encuentran en constante variación, el currículo también debe ser flexible, tomando en cuenta el criterio de (Robotton. I., 2012): "se ubique en los temas ambientales con proximidad geográfica tanto a las personas que elaboran los planes de estudio, como a las personas a las que se involucra, en el desarrollo del mismo".

Dentro del diseño de los diferentes PEA (Programa de Estudio de Asignatura) las carreras deben enfocar en su currículum los temas que desde cada perspectiva tributen a brindar alternativas de solución a los problemas ambientales que vive su provincia, su ciudad y su país.

El nuevo documento curricular de la (Básica, 2006) tiene como objetivo desarrollar la condición humana y preparar para la comprensión, para lo cual el accionar educativo se orienta a la formación de ciudadanos que practiquen valores que les permitan interactuar con la sociedad con respeto, responsabilidad, honestidad y solidaridad, aplicando los principios del Buen Vivir. Este último, está presente en la educación ecuatoriana como el principio rector de la educación y como hilo conductor de los ejes transversales que forman parte de la formación de valores. Es así que el eje transversal que versa sobre la protección del medio ambiente busca fomentar la interpretación de los problemas medioambientales y sus implicaciones en la supervivencia de las especies, la interrelación del ser humano con la naturaleza y las estrategias para su conservación y protección en los y las estudiantes. Con este propósito, el (Ministerio de educación, 2014) promueve el Proyecto Escuelas del Buen Vivir, en el que se insta a las instituciones educativas a alcanzar dicha acreditación, de modo que se conviertan en espacios que generen e implementen una cultura del Buen Vivir en la comunidad educativa. Este proceso es complementario al modelo pedagógico regular. A la vez que estas fomentan un cambio de actitud e inciden en las prácticas relacionadas con el cuidado del entorno natural y social y promueven valores y conductas orientadas a la equidad, la inclusión, la interculturalidad y la participación de la comunidad 
educativa, deben trabajar en líneas de acción para la implementación de prácticas del Buen Vivir que les permita, en tal sentido, acreditarse como: escuelas verdes, seguras, saludables y democráticas. La Universidad Metropolitana del Ecuador, desde la carrera de Ciencias de la Educación presta atención al problema ambiental, en su rol de formar profesionales para el sector educacional con la responsabilidad de formación de niños, adolescentes y jóvenes para la sociedad. Los hombres y mujeres que vivirán y deberán conducir al planeta a un mundo mejor, con menos contaminación, calidad de aire, agua potable, la protección de los bosques y biodiversidad. La carrera ha implementado acciones en la respuesta a las exigencias de la sociedad tales como:

Realizar un diagnóstico y pronóstico de la cultura ambiental en la carrera, en directivos, docentes y estudiantes.

Perfeccionar los Programas de estudio de las asignaturas de Ecogestión y Educación ambiental, por ser materias que, desde sus fundamentos científico pedagógicos, pueden dar respuesta a la solución de los problemas ambientales.

Sistematizar los procesos de formación ambiental, inicial y continua de los profesionales de la educación para incorporar la educación ambiental desde la perspectiva del desarrollo sostenible al desempeño profesional pedagógico ambiental.

Incorporar al desarrollo curricular de las estrategias didácticas para un desempeño profesional pedagógico ambiental en las carreras de educación y en la clase como vía principal del proceso docente educativo, así como en las tareas extracurriculares.

Realizar los estudiantes en sus horas de trabajo autónomo visitas a parques ecológicos, vinculación con tareas relacionadas con el medio ambiente, campañas de reciclaje, entre otras.

Diseñar actividades en las prácticas pre-profesionales dirigidas a la educación ambiental, tales como: siembra de árboles y plantas, así como el control de desechos sólidos.

Crear proyectos de investigación y de vinculación que aborden la educación ambiental en función de la formación de una cultura integral.

Contemplar en el perfil del profesional la formación de líderes comunitarios y elementos multiplicadores del sentimiento de conservación, mejoramiento y protección del medio ambiente. 


\section{Metodología}

En el presente trabajo se realizó un estudio descriptivo "los estudios descriptivos sirven para analizar cómo es y se manifiesta un fenómeno y sus componentes" (Hernández, 1997).

En relación a los métodos utilizados fue el análisis documental de la institución educativa: Proyecto estratégico institucional, PEI, Plan operativo anual POA, Programas de estudio de la asignatura PEA de Educación ambiental y Ecogestión donde se destacan las políticas de protección al medio ambiente, de carácter innovador, que constituyen el eje donde se articula toda la actuación educativa acorde con la diversidad.

\section{Resultados y discusión}

Aplicación de las acciones en la carrera de ciencias de la Educación.

Perfeccionamiento del PEA de la asignatura Educación ambiental en contenidos de actualidad, más ajustados a la problemática actual y contextualizados a la región y país.

Realización de 5 trabajos que abordan la temática; así como la socialización en eventos internacionales en correspondencias con proyectos de investigación y vinculación con la sociedad, se destaca "Cuidemos el planeta Tierra" y Medidas para el cuidado del medio ambiente.

Se desarrolló el proyecto de vinculación con la sociedad "Fomentando una cultura de Reciclaje" con la participación de 14 docentes de la escuela, 3 autoridades del plantel, 112 estudiantes de la escuela "Asia Laura" y 224 padres y madres de familia; de conjunto con 8 estudiantes y 4 docentes de la carrera desarrollando un conjunto de tareas que contribuyeron a la formación de una cultura de reciclaje y a elevar el nivel de concientización de docentes, directivos, estudiantes, padres y madres de familia acerca de la necesidad de cuidar y proteger el medio ambiente, tales como:

Identificar los fundamentos teóricos sobre la educación ambiental y en particular la cultura de reciclaje.

Conocer el proceso metodológico para desarrollar productos reciclados a partir de envases plásticos. 
Presentar Feria de manualidades con envases plásticos reciclados.

La realización del proyecto permitió conocer que la decisión de reutilizar y reciclar algunos elementos, como es el caso de botellas plásticas, también permite reducir el costo de producir productos de manera significativa.

\section{Conclusiones}

Luego de haber realizado el análisis de la problemática podemos concluir que:

Las conceptualizaciones teóricas relacionados con la formación de una cultura ambiental permitieron determinar: principios, categorías y acciones a desarrollar para responder al problema planteado.

La formación en educación ambiental debe estar orientada a: tomar conciencia de la problemática que sucede en su entorno global, a través de una formación de valores sociales, con una motivación de querer ser parte activa en la protección del medio ambiente, asumiendo las actitudes necesarias, con una responsabilidad que garantice las medidas para resolver los problemas del medio ambiente, para luego de ello evaluar los logros alcanzados y los errores corregirlos.

Los programas de estudio de Educación ambiental y Ecogestión, deben ser diseñados con las fundamentaciones científicas de manera que tributen a todas las carreras, la aplicación práctica del currículo debe estar apoyada con actividades prácticas que puedan dar respuesta a la solución de los problemas ambientales, tales como visitas a parques ecológicos, vinculación en tareas relacionadas con el medio-ambiente, campañas de reciclaje y mingas de limpieza.

Es indispensable una educación que forme en labores ambientales, dirigida tanto a las generaciones jóvenes como a los adultos, que preste la debida atención al sector de la población menos privilegiada, inspirada en el sentido de su responsabilidad en cuanto a la protección y mejoramiento del medio, por lo cual las prácticas pre-profesionales y vinculación con la sociedad de las diversas carreras deben destacar la importancia del cuidado y protección del medio ambiente. 


\section{Referencias Bibliográficas}

Básica, E. G. (2006). Documento curricular. Actualización y fortalecimiento curricular, Quito. Obtenido de http://educacion.gob.ec/actualizacion-curricular

Bermúdez.O. (2005). El diálogo de saberes y educación ambiental. Bogotá, Colombia: Universidad Nacional de Colombia.

García. E., R. y. (1996). La Educación Ambiental: bases éticas, conceptuales y metodológicas. Granada, España: Aljibe.

Hernández, B. C. (1997). Metodología de la investigación. Colombia: Panamericana, formas e impresos.

Ibarra. G. (1998). Escenarios de la Educación Ambiental en la educación. Revista Educación y Ciencia, 18, 65-78.

Maya. A. (2003). La diosa Némesis: Desarrollo sostenible o cambio cultural. Cali: Corporación Universitaria Autónoma de Occidente.

Ministerio de educación. (2014). Proyecto Escuelas del Buen Vivir. Obtenido de http://educacion.gob.ec/proyecto-escuelas-del-buen-vivir

Novo. M. (1995). La Educación Ambiental: bases éticas, conceptuales y metodológicas. Revista Iberoamericana de Educación $\mathrm{N}^{\circ} 11$.

ONU. (1992). Declaración de Río sobre el Medio ambiente y el desarrollo. Declaración, Organización de las Naciones Unidas para la educación, la ciencia y la cultura UNESCO, Departamento de asuntos económicos y sociales.

Organización de las Naciones Unidas para la Educación, 1. C. (1994). Tendencias de la educación ambiental a partir de la Conferencia de Tbilisi. Bilbao.

República del Ecuador. (2008). Constitución de la República del Ecuador. Registro Oficial.

Robotton. I. (2012). Investigación y desarrollo profesional en educación ambiental. Revista Investigación y Educación ambiental. Bogotá, Colombia: Stilo impresiones. 
Tamayo. M. (1999). La investigación. (ICFES, Ed.) Bogotá, Colombia: Stilo impresiones.

UNESCO-PNUMA. (1987). Programa de las Naciones Unidas para el Medio Ambiente. Congreso sobre educación y formación ambiental, ONU, Organización de las Naciones Unidas para la educación, la ciencia y la cultura.

Vega. R. (1999). Historia. Conocimiento y enseñanza. La cultura popular y la historia oral en el medio escolar. Bogotá: Antropos.

\section{Anexos}

Gráfico 1. Participación de estudiantes en tareas de reciclaje

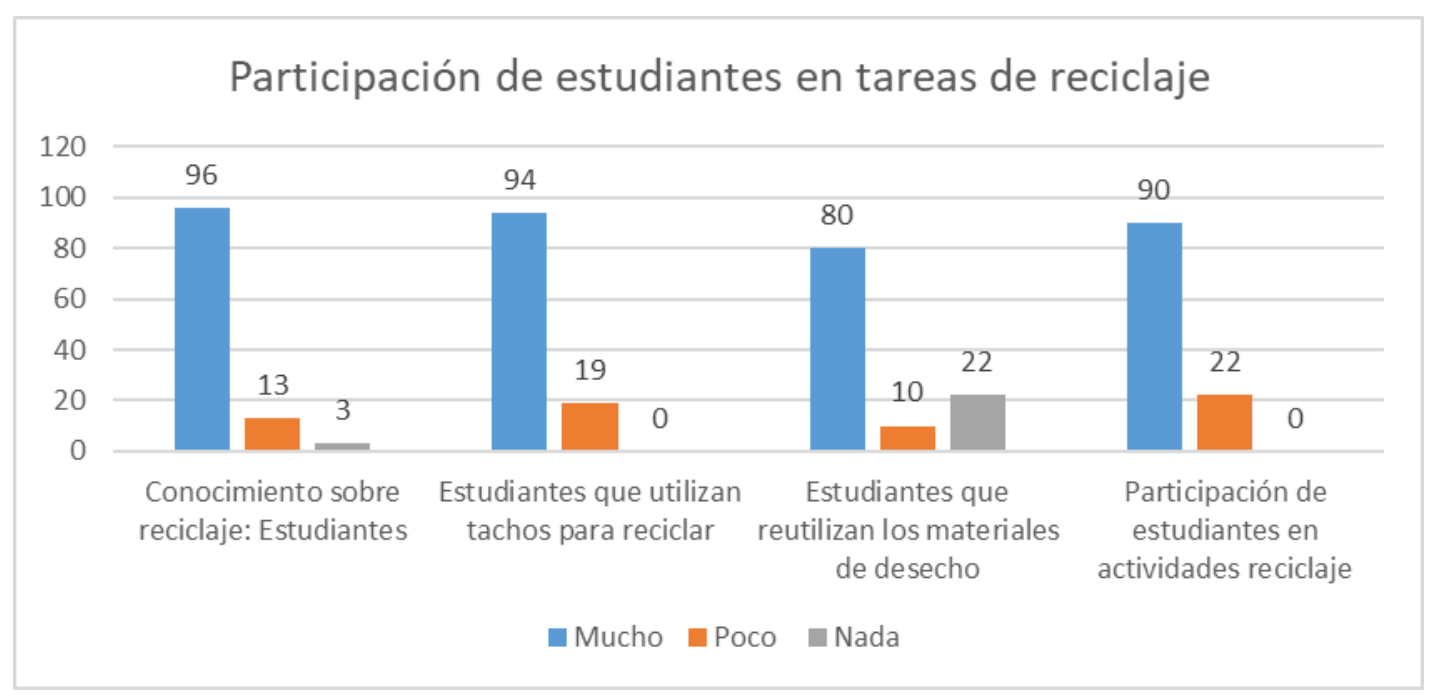

Fuente. Autora. Margarita Luque E. Ecuador,2017 
Gráfico 2. Participación de maestros en tareas de reciclaje

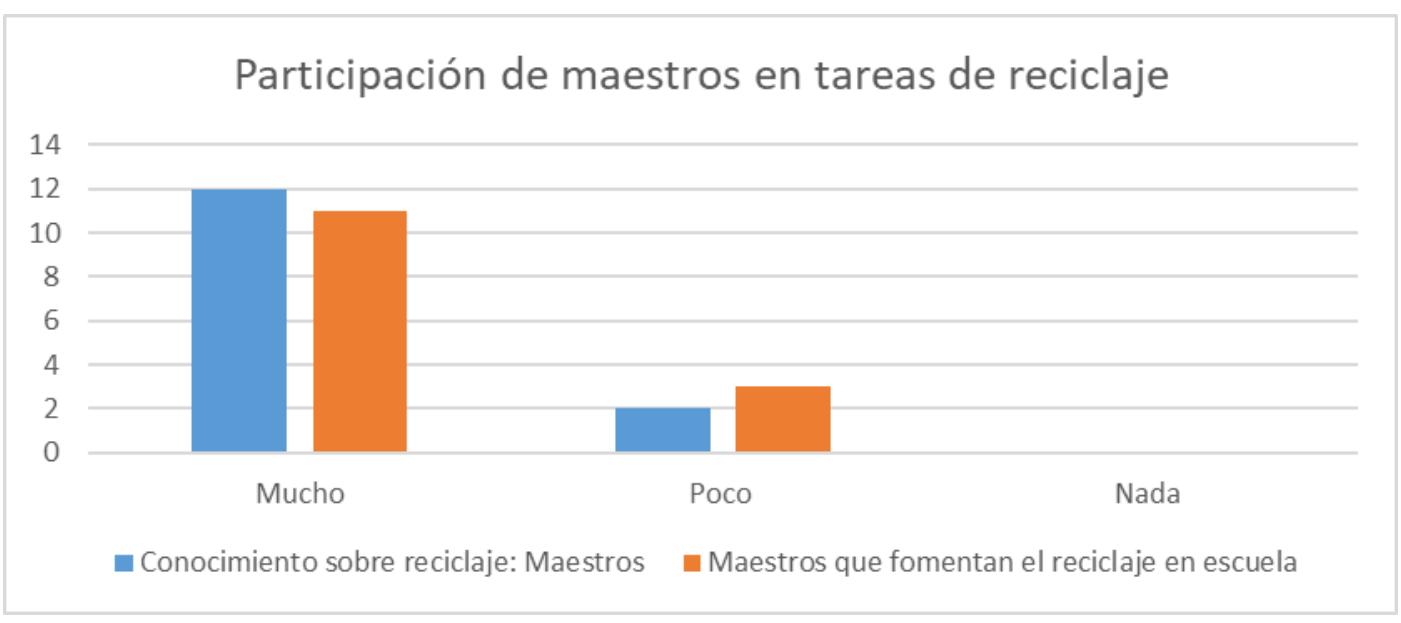

Fuente. Autora. Margarita Luque E. Ecuador. 2017

Gráfico 3. Participación de padres de familia en mingas de limpieza

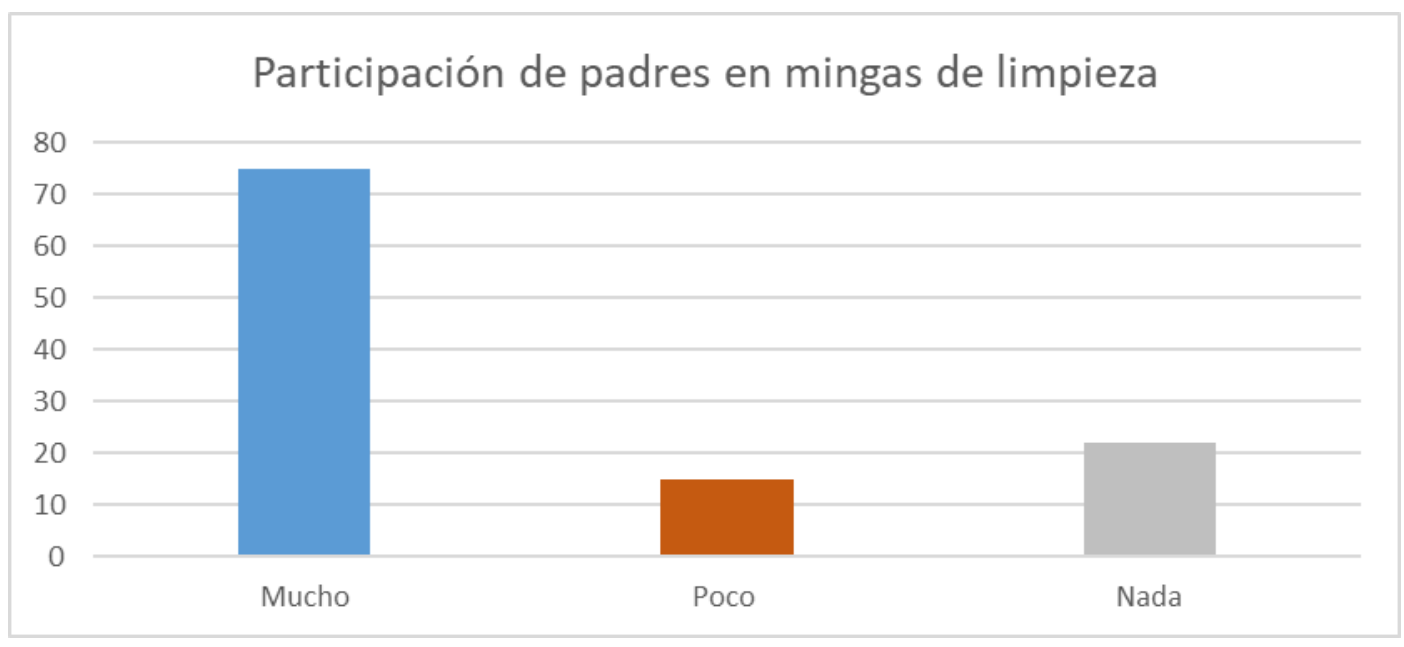

Fuente. Autora. Margarita Luque E. Año. 2017 